\title{
Tensão Superficial Estática de Misturas em Tanque de Glyphosate + ChLORIMURON-ETHYL IsOladas OU Associadas COM AdJUVAnTES $^{1}$
}

\author{
Static Superficial Tension of Glyphosate and Chlorimuron-Ethyl Tank Mixtures with or without \\ Adjuvants
}
MACIEL, C.D.G. ${ }^{2}$, GUERRA, N. ${ }^{3}$, OLIVEIRA NETO, A.M. ${ }^{3}$, POLETINE, J.P. ${ }^{2}$, BASTOS, S.L.W. ${ }^{3}$ e DIAS, N.M.S. ${ }^{3}$

\begin{abstract}
RESUMO - Objetivou-se neste estudo avaliar a tensão superficial estática, o pH e a produção da espuma de misturas em tanque de glyphosate + chlorimuron-ethyl, associadas ou não com adjuvantes. Dois trabalhos foram realizados, sendo a primeira etapa desenvolvida em delineamento inteiramente casualizado com seis tratamentos e 20 repetições, representados por soluções dos herbicidas glyphosate $\left(540,0\right.$ g e.a. ha $\left.{ }^{-1}\right)$ e chlorimuron-ethyl (7,5 g ha h $\left.^{-1}\right)$, isolados e em mistura em tanque nas formulações: Polaris ${ }^{\circledR}$, Roundup Ready ${ }^{\circledR}$, Classic $^{\circledR}$, Polaris $^{\circledR}+$ Classic $^{\circledR}$, Roundup Ready ${ }^{\circledR}+$ Classic $^{\circledR}$ e testemunha (água). Na segunda etapa foram estudados em DIC 70 tratamentos e 20 repetições, em fatorial 2 x 5 x 7 , constituído por duas misturas de glyphosate com chlorimuron-ethyl (Polaris ${ }^{\circledR}+$ Classic $^{\circledR}$ e Roundup Ready ${ }^{\circledR}+$ Classic $^{\circledR}$ ), cinco adjuvantes (Joint Oil ${ }^{\circledR}$, Nimbus $^{\circledR}$, Assist $^{\circledR}$, Natur' Oil ${ }^{\circledR}$ e Agr' Óleo ${ }^{\circledR}$ ) e sete doses dos adjuvantes (0,000; 0,031; 0,062; 0,125; 0,250; 0,500 e 1,000\% de v/v). As soluções de Polaris ${ }^{\circledR}$ e Roundup Ready ${ }^{\circledR}$, isoladas ou em misturas com Classic ${ }^{\circledR}$, caracterizaram-se por tensões superficiais estáticas de 43,2 e 35,9 $\mathrm{mN} \mathrm{m}^{-1}$ e $\mathrm{pH}$ médios em torno de 4,5 a 4,6, respectivamente. As misturas de Polaris ${ }^{\circledR}+$ Classic $^{\circledR}$, Roundup Ready ${ }^{\circledR}+$ Classic $^{\circledR}$ e Classic $^{\circledR}$ apresentaram a maior quantidade e persistência da espuma ao longo do tempo, quando comparadas às formulações de Polaris ${ }^{\circledR}$ e Roundup Ready ${ }^{\circledR}$. Os adjuvantes promoveram redução da tensão superficial quando associados às misturas de Polaris ${ }^{\circledR}+$ Classic $^{\circledR}$ e Roundup Ready ${ }^{\circledR}$ + Classic ${ }^{\circledR}$, caracterizando-se em termos de eficiência pela ordem decrescente: Nimbus ${ }^{\circledR}$

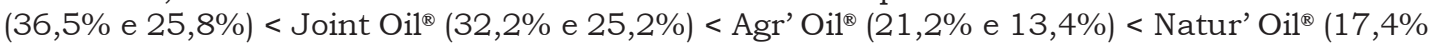
e $10,6 \%)<$ Assist $^{\circledR}(8,5 \%$ e $10,6 \%)$. Os adjuvantes promoveram redução da quantidade e persistência da espuma das misturas de Polaris ${ }^{\circledR}+$ Classic $^{\circledR}$ e Roundup Ready ${ }^{\circledR}+$ Classic $^{\circledR}$, com pequenas variações dos niveis médios de $\mathrm{pH}$ das soluções inferiores a 0,5.
\end{abstract}

Palavras-chave: $\mathrm{pH}$, espuma, herbicida, estabilidade.

\begin{abstract}
This work aimed to evaluate static superficial tension, $p H$ and foam production in glyphosate + chlorimuron-ethyl tank mixtures with or without adjuvants. The experiment consisted of two stages, with the first stage being arranged in a complete randomized design, with six treatments

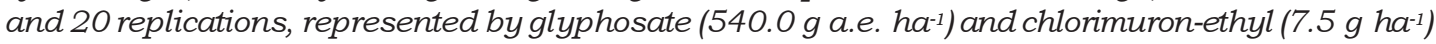
solutions, isolated and in tank mixtures in the following formulations: Polaris ${ }^{T M}$, Roundup Ready ${ }^{T M}$, Classic ${ }^{T M}$, Polaris ${ }^{T M}+$ Classic $^{T M}$, Roundup Ready ${ }^{T M}+$ Classic $^{T M}$ and check (water). The second stage was also arranged in a complete randomized design, with 70 treatments and 20 replications, in a $2 \times 5 \times 7$ factorial scheme constituted by two mixtures of glyphosate with chlorimuron-ethyl (Polaris $^{T M}+$ Classic $^{T M}$ and Roundup Ready ${ }^{T M}+$ Classic $^{T M}$ ), 5 adjuvants (Joint Oil ${ }^{T M}$, Nimbus ${ }^{T M}$, Assist ${ }^{T M}, N_{\text {atur' Oil }}{ }^{T M}$ and Agr' Óleo ${ }^{T M}$ ) and 7 adjuvant doses (0.000; 0.031; 0.062; 0.125; 0.250; 0.500 and $1.000 \%$ of $v / v$ ). Polaris ${ }^{T M}$ and Roundup Ready ${ }^{T M}$ solutions, isolated or in tank mixtures with Classic ${ }^{T M}$, characterized by static superficial tensions of 43.2 and $35.9 \mathrm{mN} \mathrm{m}^{-1}$ and medium
\end{abstract}

Recebido para publicação em 18.5.2009 e na forma revisada em 3.9.2010.

2 Professor, Dr., do Dep. de Fitotecnia da Escola Superior de Agronomia de Paraguaçu Paulista - ESAPP/FUNGE, $19700-000$ Paraguaçu Paulista-SP; <macielconsultoria@hotmail.com>; ${ }^{3}$ Acadêmicos de Agronomia da Escola Superior de Agronomia de Paraguaçu Paulista-SP

Planta Daninha, Viçosa-MG, v. 28, n. 3, p. 673-685, 2010 
pHs, around 4.5 to 4.6, respectively. Polaris ${ }^{T M}+$ Classic $^{T M}$, Roundup Ready ${ }^{T M}+$ Classic ${ }^{T M}$ and Classic ${ }^{T M}$ solutions showed the highest foam quantity and persistence during the time, compared to Polaris ${ }^{T M}$ and Roundup Ready ${ }^{T M}$ mixtures. Adjuvants promoted reduction of static superficial tension when associated with Polaris ${ }^{T M}+$ Classic $^{T M}$ and Roundup Ready ${ }^{T M}+$ Classic $^{T M}$ mixtures, being characterized in efficiency by this decreasing order: Nimbus ${ }^{T M}(36.5 \%$ and $25.8 \%)<$ Joint Oil $^{T M}$ (32.2\% and 25.2\%) < Agr' Oil TM $(21.2 \%$ and $13.4 \%)<$ Natur' Oil ${ }^{\text {TM }}(17.4 \%$ and $10.6 \%)<$ Assist ${ }^{T M}(8.5 \%$ and $10.6 \%)$. Adjuvants promoted reduction of foam quantity and persistence in the Polaris ${ }^{T M}+$ Classic $^{T M}$ and Roundup Ready ${ }^{T M}+$ Classic ${ }^{T M}$ mixtures, with small variations in the medium levels of $p H$ of the solutions lower than 0.5 .

Keywords: $\mathrm{pH}$, foam, herbicide, stability.

\section{INTRODUÇÃO}

O controle de plantas daninhas é uma prática de elevada importância para obtenção de alto rendimento em qualquer exploração agrícola e tão antiga quanto a própria agricultura. Atualmente, na cultura da soja RR, o uso de glyphosate com herbicidas seletivos em mistura em tanque tem sido uma alternativa adotada, apesar de não registrada no Ministério da Agricultura, Pecuária e Abastecimento (MAPA) para controle de espécies problemáticas, como Commelina spp. e Ipomoea spp. Entretanto, misturas em tanque de glyphosate com herbicidas latifolicidas têm frequentemente sido caracterizadas por sintomas visuais de fitointoxicação na cultura da soja, onde eles são ainda mais intensos quando utilizados adjuvantes na calda de aplicação.

A importância do uso de adjuvantes em otimizar a atividade de muitos herbicidas de pós-emergência atualmente está bem reconhecida e pode representar economia na aplicação, pela possibilidade do uso de doses reduzidas (Sherrick et al., 1986; Carbonari et al., 2004). Segundo Theisen et al. (2004), a adição de adjuvantes melhora o ambiente da calda de pulverização e as condições para a proteção e absorção dos herbicidas, uma vez que boa parte das pulverizações com herbicidas não apresenta bons resultados sem o acréscimo de algum tipo de adjuvante. Roman et al. (2007) mencionam os surfatantes como uma categoria dos adjuvantes, cuja função seria melhorar a emulsão, a dispersão, o espalhamento, o molhamento ou outras propriedades de um líquido ou solução, por meio da modificação de suas propriedades de superficie. Entretanto, Miller \& Butler Ellis (2000) e Cunha et al. (2003) relatam que os adjuvantes podem alterar o padrão das gotas da pulverização devido a mudanças nas propriedades do líquido, podendo influenciar tanto o seu processo de formação como o comportamento em relação ao alvo, alterando o risco potencial de deriva da aplicação.

Silva et al. (2006) relataram que a tensão superficial é a força que existe na superficie dos líquidos. Essa tensão se deve às fortes ligações intermoleculares, as quais dependem das diferenças elétricas entre as moléculas, e pode ser definida como a força por unidade de comprimento que duas camadas superficiais exercem uma sobre a outra.

O efeito da tensão superficial está presente em diversos segmentos industriais, como os de cosméticos, de materiais de limpeza, de tintas e de produtos fitossanitários. $\mathrm{Na}$ área dos produtos fitossanitários, o seu efeito é fundamental para o desenvolvimento de formulações e para a eficácia nas aplicações em campo. Nas formulações, é importante a presença de compostos que reduzem a tensão superficial, facilitando o contato entre os diversos componentes de um produto formulado, promovendo a diluição do produto em água e aumentando a estabilidade da solução obtida (Bianco, 1985). Atualmente, são comercializados diversos tipos de herbicidas, diferenciandose pela concentração do ingrediente ativo e pela presença ou ausência de surfatantes em sua composição. Para Schönherr et al. (1991), os surfatantes, como agentes modificadores das características fisico-químicas de soluções, 
causam alterações na adesão, velocidade de espalhamento, área de molhamento e retenção das gotas pulverizadas sobre as superficies foliares.

No Brasil, devido ao pequeno número de informações sobre o comportamento da interação entre adjuvantes e herbicidas, assim como se eles apresentam características surfatantes, é importante a compreensão do comportamento da tensão superficial entre outras características físico-químicas das soluções. Nesse contexto, essas informações tendem a reforçar a necessidade de otimizar o desempenho da água na aplicação de agrotóxicos, preparando, para isso, soluções de herbicidas que visam obter a máxima eficiência e o menor risco para os profissionais e o meio ambiente, envolvidos no processo de aplicação.

Objetivou-se com este trabalho avaliar a tensão superficial estática, o pH e a formação de espuma de misturas em tanque de glyphosate + chlorimuron-ethyl, isoladas ou associadas com adjuvantes.

\section{MATERIAL E MÉTODOS}

O trabalho foi realizado nos laboratórios da Escola Superior de Agronomia de Paraguaçu Paulista-SP (FUNGE/ESAPP), entre os meses de setembro de 2007 e janeiro de 2008. Dois experimentos foram desenvolvidos, em delineamento experimental inteiramente casualizado, para avaliar as características tensão superficial estática, pH e produção de espumas (formação e estabilidade) de caldas de pulverização constituídas pelos herbicidas glyphosate e chlorimuron-ethyl, de forma isolada ou associada com adjuvantes.

A primeira etapa foi desenvolvida com seis tratamentos e 20 repetições, com os herbicidas glyphosate (540 g e.a. ha-1) e chlorimuron-ethyl (7,5 $\left.\mathrm{g} \mathrm{ha}^{-1}\right)$, isolados e em mistura em tanque, nas respectivas formulações comerciais e doses: Polaris ${ }^{\circledR}$ (1.500 L ha-1), Roundup Ready ${ }^{\circledR}$ (1.125 L ha-1), Classic $^{\circledR}\left(30 \mathrm{~g} \mathrm{ha}^{-1}\right)$, Polaris ${ }^{\circledR}+$ Classic $^{\circledR}(1.500 \mathrm{~L}$ + 30 g ha $^{-1}$ ), Roundup Ready ${ }^{\circledR}+$ Classic $^{\circledR}(1.125 \mathrm{~L}$ + $30 \mathrm{~g} \mathrm{ha}^{-1}$ ) e testemunha (água de poço artesiano), para determinação da tensão superficial da solução. Para avaliação do pH e formação de espuma das soluções, foram utilizadas quatro repetições, sendo as unidades experimentais erlenmeyers de $250 \mathrm{~mL}$, onde as leituras de estabilidade da formação de espuma foram realizadas até três horas após preparo e agitação das soluções. Na segunda etapa foram estudados 70 tratamentos e 20 repetições, em esquema fatorial $2 \times 5 \times 7$, constituído por duas misturas em tanque de glyphosate+chlorimuron-ethyl (Polaris $^{\circledR}+$ Classic $^{\circledR}$ e Roundup Ready ${ }^{\circledR}+$ Classic ${ }^{\circledR}$ ), cinco adjuvantes (Joint Oil ${ }^{\circledR}$, Nimbus $^{\circledR}$, Assist $^{\circledR}$, Natur' Oil ${ }^{\circledR}$ e Agr' Óleo ${ }^{\circledR}$ ) e sete doses dos adjuvantes (0,000; 0,031; 0,062; 0,125; 0,250; 0,500; e 1,000\% de v/v). Para avaliação do $\mathrm{pH}$ e formação de espuma das soluções na presença dos adjuvantes, foi utilizado o mesmo número de repetições e procedimentos da primeira etapa.

As tensões superficiais estáticas das soluções foram avaliadas segundo metodologia descrita por Mendonça et al. (1999), medindo-se a massa das gotas formadas na extremidade de uma bureta posicionada dentro de uma balança analitica (precisão de 0,01 mg), em tempo determinado entre 25 e 30 segundos. Cada conjunto de três gotas pesadas correspondeu a uma repetição; para evitar perdas por evaporação, utilizou-se para captura das gotas óleo vegetal em béquer de $25 \mathrm{~mL}$, posicionado abaixo da ponta da bureta e sobre o prato da balança. $\mathrm{O}$ pH das soluções foi avaliado com auxílio de peagômetro de bancada digital aos 0,5 ; $1 ; 0 ; 1,5 ; 2,0 ; 2,5 ;$ e 3,0 horas após agitação inicial única e constante, em agitador mecânico do tipo coqueteleira metálica, durante $10 \mathrm{~min}$ em rotação de $200 \mathrm{rpm}$, e a altura do volume de espuma e sua estabilidade, conforme metodologias adaptadas de Embrapa (1997), Coupland \& Robinson (1992) e Mendonça et al. (2004). Nas avaliações de altura do volume de espuma utilizouse régua graduada, além de serem descritos qualitativamente os possiveis efeitos de sedimentação, floculação, alteração e/ou formação de fases das soluções estudadas. A temperatura média do ambiente dos laboratórios foi registrada entre $25{ }^{\circ} \mathrm{C} \pm 3{ }^{\circ} \mathrm{C}$.

Os dados de $\mathrm{pH}$ e altura do volume de espuma foram submetidos à análise de variância pelo teste $\mathrm{F}$, e as suas médias, 
comparadas pelo teste de Tukey a $5 \%$ de probabilidade. Para as avaliações de tensão superficial estática, os dados originais foram submetidos a análises de variância e regressão, com o auxílio do programa SAS, ajustando-os ao modelo de Mitscherlich simplificado, conforme:

Mitscherlich original: $Y=A(1-10-\mathrm{C}(X+B))$

Mitscherlich utilizado na forma simplificada: $Y=$ Tágua $-A(1-10-C(X+B))$

em que $Y=$ tensão superficial $\left(\mathrm{mN} \mathrm{m}^{-1}\right) ; A=$ assintota horizontal máxima no modelo original; $C=$ concavidade da curva; corresponde à eficiência do adjuvante quanto maior o valor desse parâmetro, mais eficiente o adjuvante será para atingir a tensão superficial mínima em menor concentração; $B=$ ponto de interceptação do eixo das abscissas; corresponde ao quanto deve ser adicionado de adjuvante para obter a mesma redução de tensão condicionada pela adição das misturas dos herbicidas, nas concentrações consideradas; Tágua = $72,6 \mathrm{~m} \mathrm{~N} \mathrm{~m}^{-1} ; X=$ concentração dos adjuvantes $(\%$ volume/volume $)$; e (Tágua $-\mathrm{A})=$ assintota horizontal no modelo simplificado; corresponde à mínima tensão superficial que o adjuvante pode alcançar a partir da concentração em que atinge esse valor de tensão superficial - mesmo quando aumentada a concentração do adjuvante, não se obtêm decréscimos nos valores de tensão.

No trabalho também foram estimados os coeficientes de micelação (concentração micelar crítica $=\mathrm{CMC}$ ) dos adjuvantes, correspondendo ao ponto de inflexão da curva da tensão superficial, em que, acima da CMC, monômeros do adjuvante em solução se agregam em estruturas coloidais denominadas micelas. A partir desse ponto, o incremento do adjuvante traz pouco benefício em relação à capacidade de este destensionar as soluções (Rosen, 1989; Buick et al.,1993). Dessa forma, para definição da $\mathrm{CMC}$, considerou-se que em baixas concentrações do adjuvante inexiste a formação de micelas e, acima da CMC, toda nova adição do adjuvante à solução será incorporada a novas micelas ou às já existentes, sem haver incremento de redução da tensão. Quanto ao cálculo do coeficiente de micelação, foi adotado um procedimento alternativo, de forma semelhante à utilizada por Montório (2001), em que a partir das curvas de tensão superficial em função das concentrações dos adjuvantes, segundo a expressão: coeficiente de micelação $=100 \mathrm{x}$ (1 - derivada de uma dada concentração/ derivada quando a concentração é nula).

\section{RESULTADOS E DISCUSSÃO}

As tensões superficiais estáticas das soluções de glyphosate Polaris ${ }^{\circledR}$ e Roundup Ready ${ }^{\circledR}$, isoladas ou em mistura com chlorimuron-ethyl (Classic ${ }^{\circledR}$ ), no experimento 1 , foram caracterizadas por niveis em torno de 43,2 e $35,9 \mathrm{mN} \mathrm{m}^{-1}$, respectivamente, não tendo sido constatada interferência do chlorimuron-ethyl sobre o glyphosate nas referidas associações. Os pHs médios das soluções de Polaris ${ }^{\circledR}$ e Roundup Ready ${ }^{\circledR}$, isoladas ou em mistura com Classic ${ }^{\circledR}$, foram mantidos em torno de 4,5 a 4,6 , respectivamente, não diferindo significativamente entre si (Figura 1).

Dessa forma, os resultados indicaram a boa compatibilidade das misturas de chlorimuron-ethyl com as formulações de glyphosate, em relação às características físico-químicas das soluções, as quais podem beneficiar aspectos de formação e deposição das gotas de pulverização caracterizadas pela redução da tensão superficial e/ou favorecer a absorção e dissociação dos herbicidas, por meio da diminuição do $\mathrm{pH}$. Para Ellis \& Tuck (1997), além da deposição, evaporação, espalhamento e escorrimento da calda nos alvos, a tensão superficial também pode alterar o diâmetro das gotas formadas pelas pontas de pulverização. McCormick (1990) relata que a intensidade das reações na calda de pulverização é variável com o pH e o tipo de íon da solução; as propriedades físico-químicas e a atividade dos herbicidas podem ser alteradas com a redução do $\mathrm{pH}$. Para esse autor, as máximas absorção e eficiência de herbicidas com caráter ácido, como o glyphosate, ocorrem em $\mathrm{pH}$ em que $50 \%$ das moléculas se encontram dissociadas. Resultados descritos por Dan et al. (2009) mencionam melhor eficácia do glyphosate no controle de Brachiaria brizantha com $\mathrm{pH}$ da calda de pulverização 


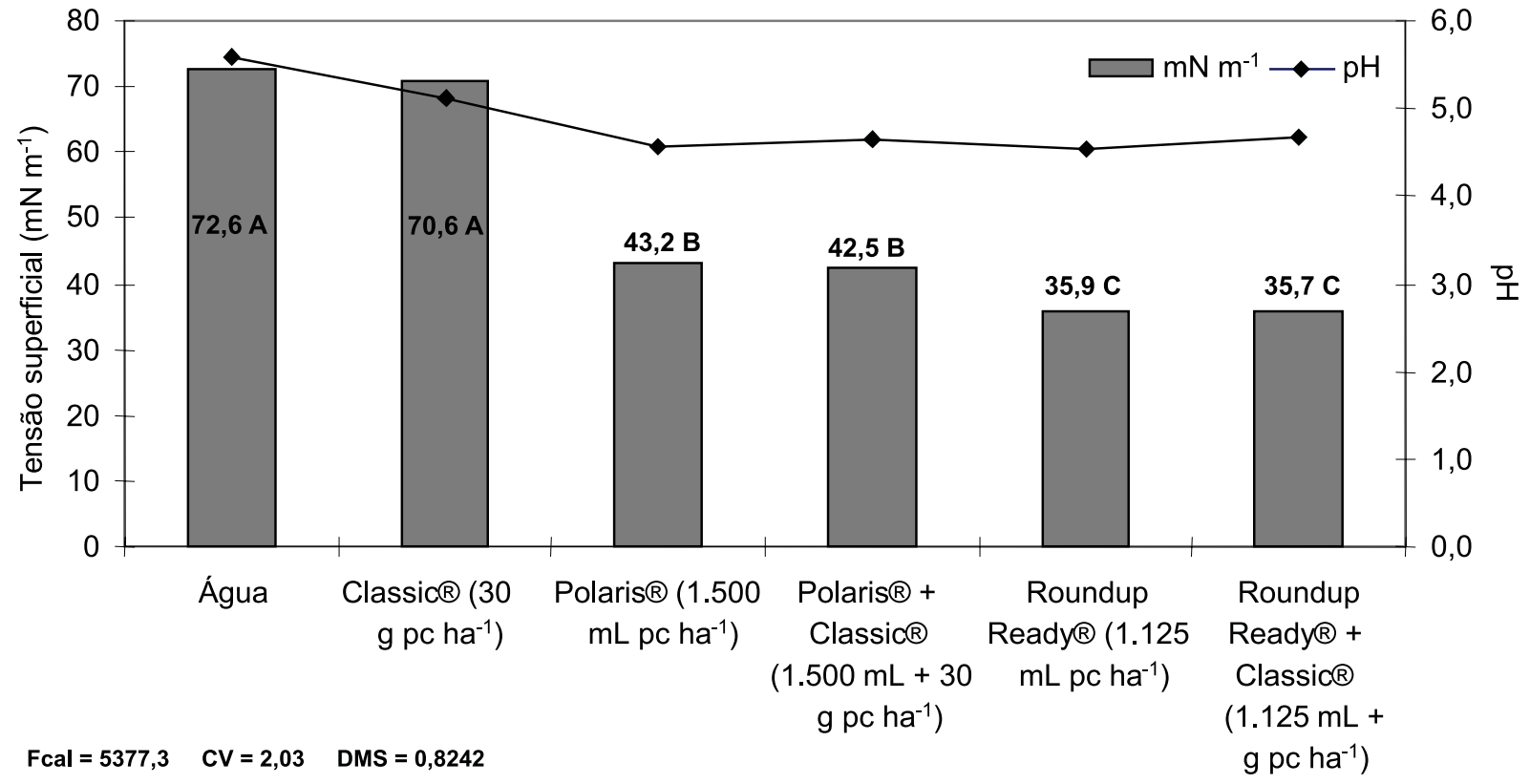

Figura 1 - Tensões superficiais estáticas e pHs das soluções com os herbicidas glyphosate (Polaris ${ }^{\circledR}$ e Roundup Ready ${ }^{\circledR}$ ) e chlorimuronethyl $\left(\mathrm{Classic}^{\circledR}\right)$, isolados e associados em misturas. As letras maiúsculas nas colunas e minúsculas nas linhas representam médias que não diferem entre si pelo teste de Tukey $(\mathrm{p}<0,05)$.

entre 3,5 e 5,5, assim como os menores niveis de mortalidade em $\mathrm{pH} 1,3$ e 9,0. Sanchotene et al. (2007) concluíram que água acidificada em $\mathrm{pH} 4,5$ potencializou a eficácia dos inibidores de ALS imazethapyr + imazapic no controle de arroz-vermelho (Oryza sativa), sugerindo que isso também é observado para o glyphosate.

Com relação à espuma formada através do preparo e agitação das soluções (Figura 2), observa-se que as soluções de Polaris ${ }^{\circledR}$ Classic $^{\circledR}$, Roundup Ready ${ }^{\circledR}+\mathrm{Classic}^{\circledR}$ e Classic $^{\circledR}$ foram as que apresentaram a maior quantidade e persistência da espuma ao longo do tempo das avaliações, quando comparadas com as formulações de glyphosate na ausência do chlorimuron-ethyl. Ao final de uma hora após preparo e agitação da solução, não foi mais constatada a presença de espuma para a formulação Roundup Ready $^{\circledR}$. Entretanto, a solução com glyphosate Polaris ${ }^{\circledR}$ ainda manteve a formação de espuma até duas horas após preparo e agitação; apesar de não diferir significativamente em quantidade, ela não apresentava espuma no referido período. Segundo Mendonça et al. (2004), a espuma formada durante o preparo

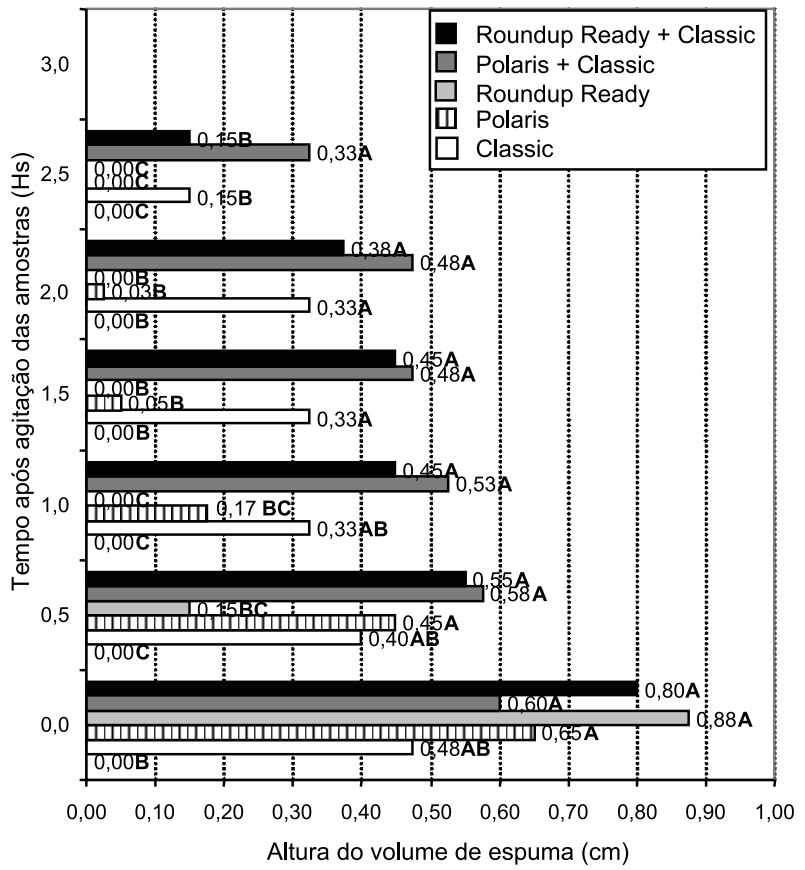

Figura 2 - Altura média do volume de espuma produzido após agitação de soluções com as formulações dos herbicidas glyphosate (Polaris ${ }^{\circledR}$ e Roundup Ready ${ }^{\circledR}$ ) e chlorimuron-ethyl $\left(\right.$ Classic $^{\circledR}$ ), isolados e associados em misturas. Colunas sob letras maiúsculas representam médias que não diferem entre si pelo teste de Tukey $(\mathrm{p}<0,05)$ 
da calda no tanque de pulverização não é uma característica desejável, uma vez que geralmente faz-se necessário interromper a adição de água quando a espuma começa a transbordar do tanque. Assim, a espuma ocupa um volume que deveria estar preenchido pela água, tornando a calda mais concentrada do que o programado, e/ou mesmo pode prolongar o tempo de preparo da calda de pulverização, ao aguardar a sua dissipação.

As curvas de tensões superficiais demonstradas para misturas entre as formulações Polaris ${ }^{\circledR}$ e Roundup Ready ${ }^{\circledR}$ com Classic ${ }^{\circledR}$ apresentaram características distintas quando associadas com as adjuvantes estudados no experimento 2 (Figuras 3 e 4). Os baixos valores do coeficiente de variação, assim como os coeficientes de determinação das curvas ajustadas, superiores a 0,93, para Polaris $^{\circledR}+$ Classic $^{\circledR}$ e Roundup Ready ${ }^{\circledR}+$ Classic $^{\circledR}$ associados a Nimbus ${ }^{\circledR}$ e Joint Oil ${ }^{\circledR}$, caracterizaram elevada precisão do método e modelo adotado (Tabelas 1 e 2). Para os adjuvantes Agr' $\mathrm{Oil}^{\circledR}$, Natur' $\mathrm{Oil}^{\circledR} \mathrm{e}^{\mathrm{e}}$ Assist ${ }^{\circledR}$, os coeficientes de determinação das curvas apresentaram-se entre 0,90 e 0,72, indicando precisão entre satisfatória e intermediária. A análise dos dados indica que a avaliação da tensão superficial através da massa de gotas produzidas por tubos capilares pode ser feita com alta precisão, corroborando os resultados de Mendonça et al. (1999), Velini et al. (2000) e Silva et al. (2006).

Além da precisão experimental observada pela adequação do método, os resultados ilustrados nas Figuras 3 e 4 também demonstraram, por meio da maior inclinação e concavidade das curvas, o melhor desempenho dos adjuvantes $\mathrm{Nimbus}^{\circledR}$ e Joint $\mathrm{Oil}^{\circledR}$ sobre Agr' Oil ${ }^{\circledR}$, Natur' Oil ${ }^{\circledR}$ e Assist ${ }^{\circledR}$ em reduzir a tensão superficial das soluções quando associados às misturas de Polaris ${ }^{\circledR}+$ Classic $^{\circledR}$ e Roundup Ready ${ }^{\circledR}+$ Classic $^{\circledR}$. Outra forma de confirmar a coerência dos resultados favoráveis às misturas associadas ao Nimbus $^{\circledR}$ e Joint Oil ${ }^{\circledR}$ é por meio da ocorrência dos menores valores do parâmetro $\mathrm{C}$ da equação de Mitscherlich simplificada descritos nas Tabelas 1 e 2 . Os menores valores da referida constante indicam a ocorrência de maior redução da tensão superficial das soluções estudadas. Dessa forma, os valores da constante $\mathrm{C}$ refletem a capacidade da solução em atingir a tensão superficial mínima em menor concentração do adjuvante utilizado, assim como correspondem ao principal indicador da eficiência do adjuvante em promover novas reduções de tensão para acréscimos unitários em suas concentrações (Silva et al., 2006).

O aumento da ação dos herbicidas pela adição de surfatantes à calda de pulverização é um resultado normalmente frequente em trabalhos científicos. Entre os aspectos favoráveis explorados dessa tecnologia encontrase a possibilidade de permitir redução de dose ou ampliar a eficácia contra espécies de difícil controle, em que o incremento de eficiência está relacionado com aumento da molhabilidade da superfície, redução de tensão superficial e ângulo de contato da gota, além do aumento da penetração cuticular (Singh \& Marck, 1993). Chung \& Kwon (1992), Mendonça et al. (1999) e Costa et al. (2005) também observaram decréscimo da tensão superficial para soluções de glyphosate associadas a surfatantes, havendo aumento da área de molhamento sobre folhas de plantas daninhas. Entretanto, Matuo \& Baba (1981) e Silva et al. (2008) verificaram escorrimento da calda de pulverização em folhas de citros e café devido à adição de adjuvantes.

É importante ressaltar que nem sempre o uso de adjuvantes estabelecerá condição favorável à deposição da pulverização, principalmente quando não são considerados os variáveis efeitos sobre o tamanho de gotas e as diferentes composições anatômicas e, principalmente, das ceras epicuticulares das plantas daninhas e culturas. Nesse contexto, o grau de sensibilidade de um herbicida pode estar associado à morfologia da superficie foliar, bem como à composição química da cera epicuticular (Procópio et al., 2003; Ferreira et al., 2005). Monquero et al. (2004) observaram que Commelina benghalensis possui maior tolerância ao glyphosate, em relação a Ipomoea grandifolia e Amaranthus hybridus, por apresentar maior proporção de hidrocarbonetos altamente hidrofóbicos na cera epicuticular. 

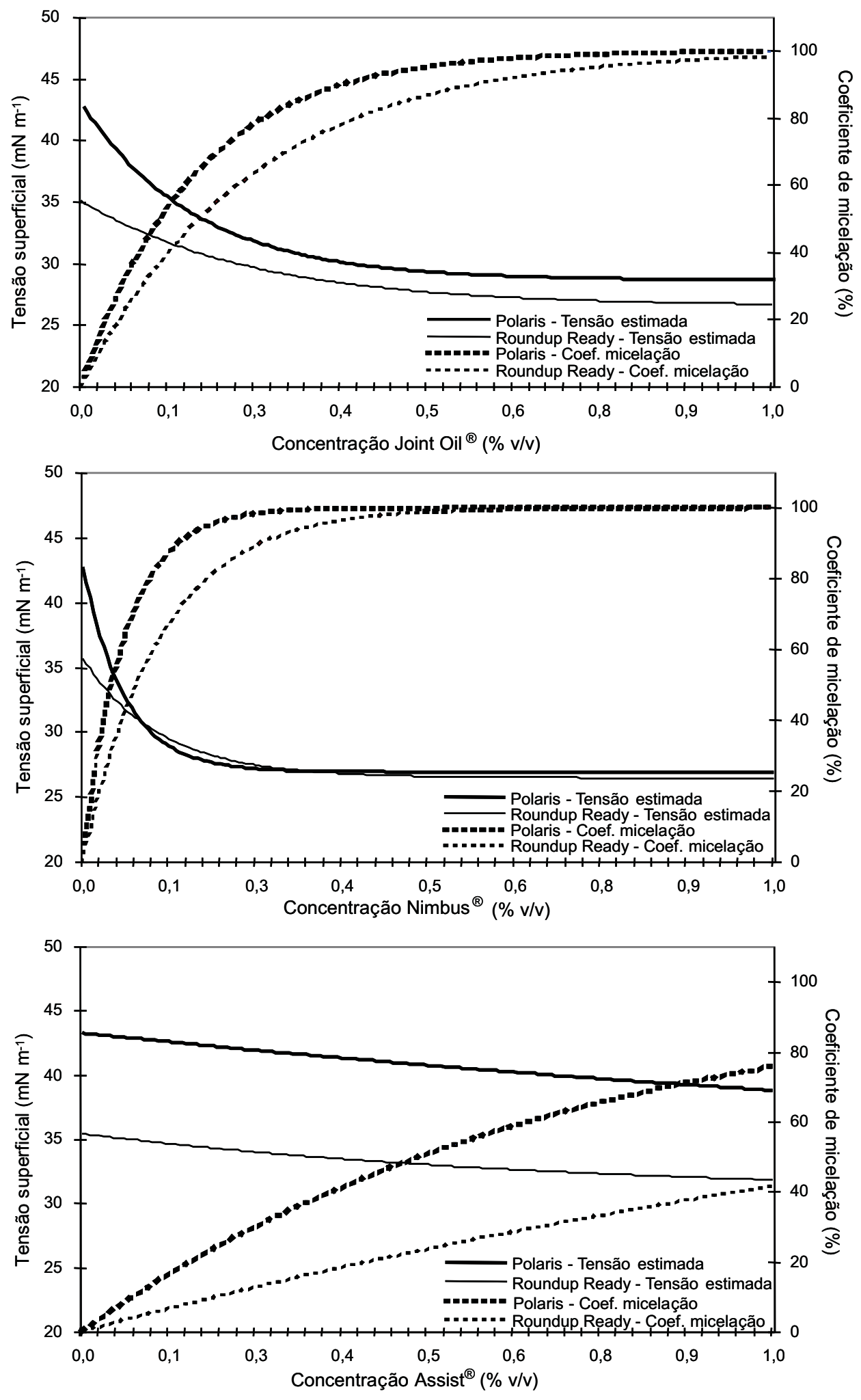

Figura 3 - Tensões superficiais e coeficiente de micelação de misturas em tanque dos herbicidas glyphosate (Polaris ${ }^{\circledR}$ e Roundup Ready $^{\circledR}$ ) e chlorimuron-ethyl $\left(\right.$ Classic $\left.^{\circledR}\right)$, em função das concentrações de adjuvantes. Equações de regressão ajustadas ao modelo de Mitscherlich simplificado. 

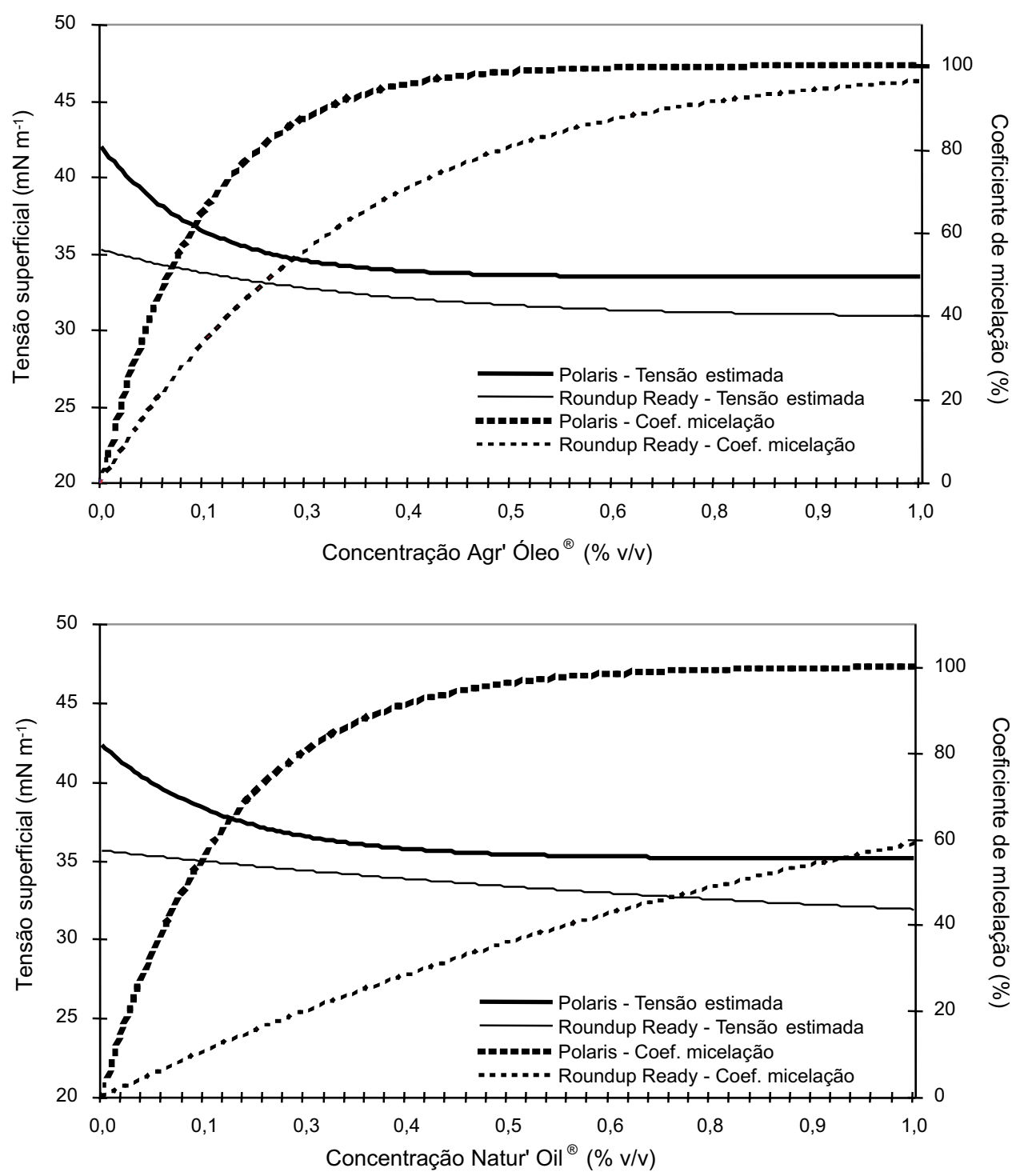

Figura 4 - Tensões superficiais e coeficiente de micelação de misturas em tanque dos herbicidas glyphosate (Polaris ${ }^{\circledR}$ e Roundup Ready $^{\circledR}$ ) e chlorimuron-ethyl $\left(\right.$ Classic $\left.^{\circledR}\right)$, em função das concentrações de adjuvantes. Equações de regressão ajustadas ao modelo de Mitscherlich simplificado.

Os niveis minimos de tensão superficiais promovidos pelos adjuvantes testados associados às misturas Polari ${ }^{\circledR}+\mathrm{Classic}^{\circledR} \mathrm{e}$ Roundup Ready ${ }^{\circledR}+\mathrm{Classic}^{\circledR}$ (Figuras 3 e 4 e Tabelas 1 e 2) podem ser dispostos na seguinte ordem decrescente, em termos de porcentagem de eficiência: Nimbus ${ }^{\circledR}(36,5 \%$ e $25,8 \%)<$ Joint $\mathrm{Oil}^{\circledR}(32,2 \%$ e $25,2 \%)<$ Agr' $\mathrm{Oil}^{\circledR}(21,2 \%$ e $13,4 \%)<\mathrm{Natur}^{\prime} \mathrm{Oil}^{\circledR}(17,4 \%$ e $10,6 \%)<$ Assist $^{\circledR}(8,5 \%$ e $10,6 \%)$. Esses resultados indicam que os adjuvantes apresentaram potencial para reduzir a tensão superficial mínima das soluções de glyphosate+ chlorimuron-ethyl, sendo, de forma geral, maior a eficácia para misturas de Polaris ${ }^{\circledR}+$ Classic ${ }^{\circledR}$, quando comparadas a Roundup Ready $^{\circledR}+$ Classic $^{\circledR}$. Dessa forma, as mesmas concentrações de ingrediente ativo de glyphosate + chlorimuron-ethyl utilizadas com as formulações comerciais de Polaris ${ }^{\circledR+}$ Classic $^{\circledR}$ e Roundup Ready ${ }^{\circledR}+$ Classic $^{\circledR}$ resultaram em diferenças entre ambas as tensões mínimas obtidas das misturas, na ordem de 0,$5 ; 2,1 ; 2,6 ; 3,2$; e $7,0 \mathrm{mN} \mathrm{m}^{-1}$, respectivamente, para os adjuvantes Nimbus ${ }^{\circledR}$, Joint $\mathrm{Oil}^{\circledR}$, Agr' $^{\circ i 1^{\circledR}, \text { Natur' }^{\prime} \mathrm{Oil}^{\circledR} \text { e Assist }}{ }^{\circledR}$. 
Tabela 1 - Resultados das análises de regressão entre as tensões superficiais de misturas em tanque de Polaris ${ }^{\circledR}+$ Classic $^{\circledR}$ e as concentrações dos adjuvantes (\%), utilizando o modelo de Mitscherlich simplificado

\begin{tabular}{|c|c|c|c|c|c|c|}
\hline \multirow[b]{2}{*}{ Modelo } & \multicolumn{6}{|c|}{$Y=72,6-A^{*}\left(1-10^{\wedge}(-C(B+X))\right)$} \\
\hline & $\begin{array}{c}\text { Polaris }^{\circledR}+ \\
\text { Classic }^{\circledR}\end{array}$ & 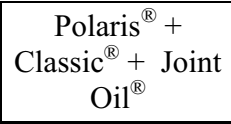 & $\begin{array}{l}\text { Polaris }^{\circledR}+ \\
\text { Classic }^{\circledR}+ \\
\text { Nimbus }^{\circledR}\end{array}$ & $\begin{array}{c}\text { Polaris }^{(B)}+ \\
\text { Classic }^{(\mathbb{B}}+ \\
\text { Assist }^{(\mathbb{B}}\end{array}$ & $\begin{array}{c}\text { Polaris }^{(\mathbb{R}}+ \\
\text { Classic }^{(\mathbb{B}}+\text { Natur' }^{\prime} \\
\text { Oil }^{\mathbb{R}}\end{array}$ & $\begin{array}{l}\text { Polaris }^{\circledR}+ \\
\text { Classic }^{\circledR}+ \\
\text { Agr' Oil }^{\circledR}\end{array}$ \\
\hline \multirow{3}{*}{$\begin{array}{l}\text { Estimativa dos } \\
\text { Parâmetros }\end{array}$} & A - & 43,8779 & 45,6411 & 40,0422 & 37,4634 & 39,0921 \\
\hline & B - & 0,1878 & 0,0638 & 2,4526 & 0,2539 & 0,1830 \\
\hline & C - & 2,6296 & 7,2086 & 0,2327 & 2,8221 & 3,6183 \\
\hline Tensão mínima & 42,5 & 28,8 & 27,0 & 38,9 & 35,1 & 33,5 \\
\hline S Q Regressão & - & $177.684,60$ & $144.889,23$ & $245.979,27$ & $14.1741,19$ & $192.816,92$ \\
\hline S Q Resíduo & - & 181,24 & 195,62 & 148,88 & 130,66 & 147,88 \\
\hline F Regressão & - & $575,40 * *$ & $1.250,63 * *$ & $11.386,32 * *$ & $287,02 * *$ & $771,12 * *$ \\
\hline $\mathrm{CV}(\%)$ & - & 2,92 & 2,44 & 0,51 & 2,28 & 1,91 \\
\hline $\mathrm{R}^{2}$ & - & 0,9526 & 0,9571 & 0,7234 & 0,8419 & 0,9002 \\
\hline
\end{tabular}

** Significativo a $1 \%$ de probabilidade; $\mathrm{Y}=$ tensão superficial $\left(\mathrm{mN} \mathrm{m}^{-1}\right) ; \mathrm{X}=$ concentração do adjuvante (\%); a, b e c são as estimativas dos parâmetros do modelo.

Tabela 2 - Resultados das análises de regressão entre as tensões superficiais de misturas em tanque de Roundup Ready ${ }^{\circledR}+$ Classic $^{\circledR} \mathrm{e}$ as concentrações dos adjuvantes (\%), utilizando o modelo de Mitscherlich simplificado

\begin{tabular}{|c|c|c|c|c|c|c|}
\hline \multirow[b]{2}{*}{ Modelo } & \multicolumn{6}{|c|}{$Y=72,6-A^{*}\left(1-10^{\wedge}(-C(B+X))\right)$} \\
\hline & $\begin{array}{c}\text { Roundup } \\
\text { Ready }^{\circledR}+ \\
\text { Classic }^{(\mathbb{B}}\end{array}$ & $\begin{array}{c}\text { Roundup Ready }{ }^{\circledR} \\
+ \text { Classic }^{\circledR}+ \\
\text { Joint Oil }^{\circledR}\end{array}$ & $\begin{array}{c}\text { Roundup Ready }{ }^{\circledR} \\
+ \text { Classic }^{\mathbb{B}}+ \\
\text { Nimbus }^{(\mathbb{R}}\end{array}$ & $\begin{array}{c}\text { Roundup Ready }{ }^{\circledR} \\
+ \text { Classic }^{\mathbb{R}}+ \\
\text { Assist }^{\mathbb{R}}\end{array}$ & $\begin{array}{c}\text { Roundup Ready }{ }^{\circledR} \\
+ \text { Classic }^{\circledR}+ \\
\text { Natur' Oil }^{\circledR}\end{array}$ & $\begin{array}{c}\text { Roundup Ready }{ }^{\circledR} \\
+ \text { Classic }^{\circledR}+ \\
\text { Agr' Oil }^{\circledR}\end{array}$ \\
\hline \multirow{3}{*}{$\begin{array}{l}\text { Estimativa dos } \\
\text { Parâmetros }\end{array}$} & A - & 46,0488 & 46,0911 & 41,908 & 43,2838 & 41,8419 \\
\hline & B - & 0,4187 & 0,1807 & 1,5387 & 2,1699 & 0,6816 \\
\hline & C - & 1,7486 & 3,8794 & 0,6137 & 0,3851 & 1,4193 \\
\hline Tensão mínima & 35,7 & 26,7 & 26,5 & 31,9 & 31,9 & 30,9 \\
\hline S Q Regressão & - & $137.514,19$ & $128.748,17$ & $163.925,89$ & $186.805,12$ & $15.6245,11$ \\
\hline S Q Resíduo & - & 88,88 & 48,05 & 48,70 & 174,04 & 80,15 \\
\hline F Regressão & - & $719,35 * *$ & $864,63 * *$ & $1.962,96 * *$ & $156,79 * *$ & $168,49 * *$ \\
\hline $\mathrm{CV}(\%)$ & - & 1,77 & 1,82 & 1,18 & 1,93 & 1,91 \\
\hline $\mathrm{R}^{2}$ & - & 0,9343 & 0,9698 & 0,8121 & 0,7410 & 0,8036 \\
\hline
\end{tabular}

** Significativo a $1 \%$ de probabilidade; $\mathrm{Y}=$ tensão superficial $\left(\mathrm{mN} \mathrm{m}^{-1}\right) ; \mathrm{X}=$ concentração do adjuvante $(\%)$; a, b e c são as estimativas dos parâmetros do modelo.

Além das tensões superficiais estimadas, as curvas dos coeficientes de micelação, em função da concentração dos adjuvantes, caracterizaram que os niveis de micelação foram desuniformes e dependentes da natureza de cada adjuvante (Figuras 3 e 4). Constata-se que os adjuvantes Nimbus ${ }^{\circledR}$, Joint Oil ${ }^{\circledR}$; Agr' Oil ${ }^{\circledR}$, Natur' $\mathrm{Oil}^{\circledR}$ e Assist $^{\circledR}$, quando em solução de Polaris ${ }^{\circledR}+\mathrm{Classic}^{\circledR}$, na concentração de, por exemplo, 0,5\% de volume/volume (v/v), apresentaram, respectivamente, 99,9, 95,2, 98,4, 96,1 e $50,7 \%$ das moléculas em micelas e $0,1 \%$, $4,8 \%, 1,6 \%, 3,9 \%$ e $49,3 \%$ das moléculas agregadas na interfase ar-líquido. Já na solução de Roundup $\operatorname{Ready}^{\circledR}+\mathrm{Classic}^{\circledR}$ a $0,5 \%$ de $\mathrm{v} / \mathrm{v}$ dos adjuvantes, observaram-se, respectivamente, 98,8, 86,6, $80,4,35,8$ e $23,5 \%$ das moléculas em micelas e $1,2 \%, 13,4 \%, 19,6 \%, 64,2 \%$ e $76,5 \%$ das moléculas agregadas na interfase ar-líquido. 
Nesse exemplo, utilizando $0,5 \%$ de $\mathrm{v} / \mathrm{v}$ dos adjuvantes, pode-se constatar que o incremento dos adjuvantes apresentou menores benefícios em destensionar as soluções para mistura de Roundup Ready ${ }^{\circledR}+$ Classic $^{\circledR}$, uma vez que para Polaris ${ }^{\circledR}+$ Classic $^{\circledR}$, com exceção do óleo mineral Assist ${ }^{\circledR}$, os coeficientes de micelação apresentaram-se superiores a $95 \%$, ou seja, muito próximos do ponto de concentração micelar crítica $(100 \%)$. Para valores muito próximos ou acima da concentração micelar crítica, em toda nova adição do adjuvante a solução será incorporada a novas micelas ou às já existentes, sem haver incremento de redução da tensão.

As concentrações dos adjuvantes estudados promoveram, de modo geral, variação do $\mathrm{pH}$ das soluções com as misturas
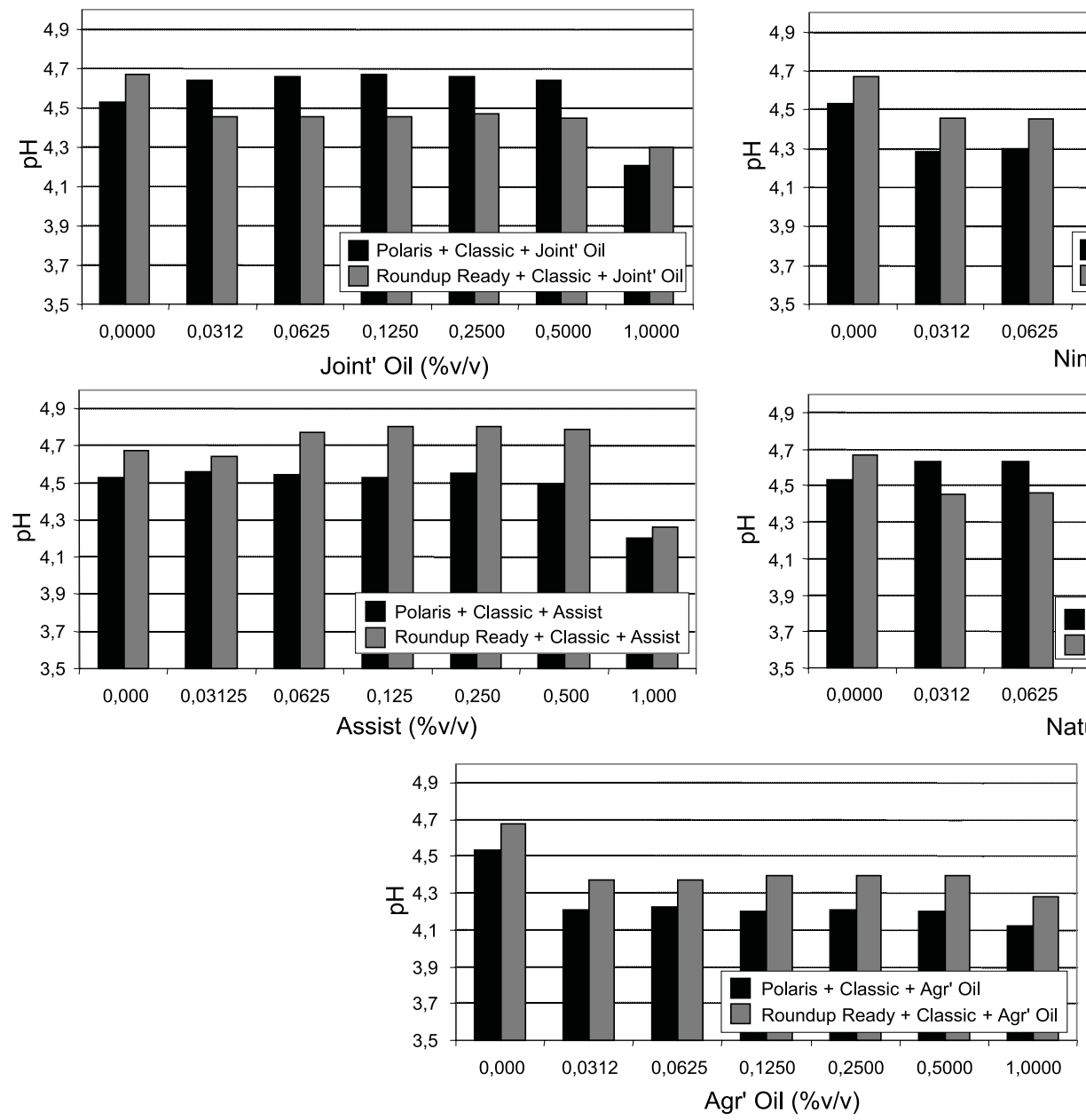

Figura 5 - Nível médio de pH das soluções imediatamente após agitação para misturas em tanque dos herbicidas glyphosate (Polaris ${ }^{\circledR}$ e Roundup Ready $\left.{ }^{\circledR}\right)$ e chlorimuron-ethyl $\left(\right.$ Classic $\left.^{\circledR}\right)$, associados com adjuvantes.

Planta Daninha, Viçosa-MG, v. 28, n. 3, p. 673-685, 2010
Polaris $^{\circledR}+$ Classic $^{\circledR}$ e Roundup Ready ${ }^{\circledR}+$ Classic $^{\circledR}$ entre 0,1 e 0,4, respectivamente (Figura 5). Em termos de redução do nível do $\mathrm{pH}$ da solução, os maiores destaques foram obtidos com Nimbus ${ }^{\circledR}$ e Agr' $^{\prime} \mathrm{Oil}^{\circledR}$, para ambas as misturas de herbicidas, e com Joint $\mathrm{Oil}^{\circledR}$ e Natur' Oil ${ }^{\circledR}$, apenas para Roundup Ready $^{\circledR}+$ Classic $^{\circledR}$.

Todos os adjuvantes apresentaram redução da quantidade e persistência da espuma das misturas de Polaris ${ }^{\circledR}+\mathrm{Classic}^{\circledR}$ e Roundup Ready $^{\circledR}+$ Classic $^{\circledR}$ (Figura 6), sendo elas mais intensas para Roundup Ready ${ }^{\circledR}+$ Classic ${ }^{\circledR}$ em termos de eficácia. Entretanto, de forma geral, a maior redução da quantidade e persistência da espuma das soluções foi constatada quando associada ao adjuvante Nimbus ${ }^{\circledR}$.
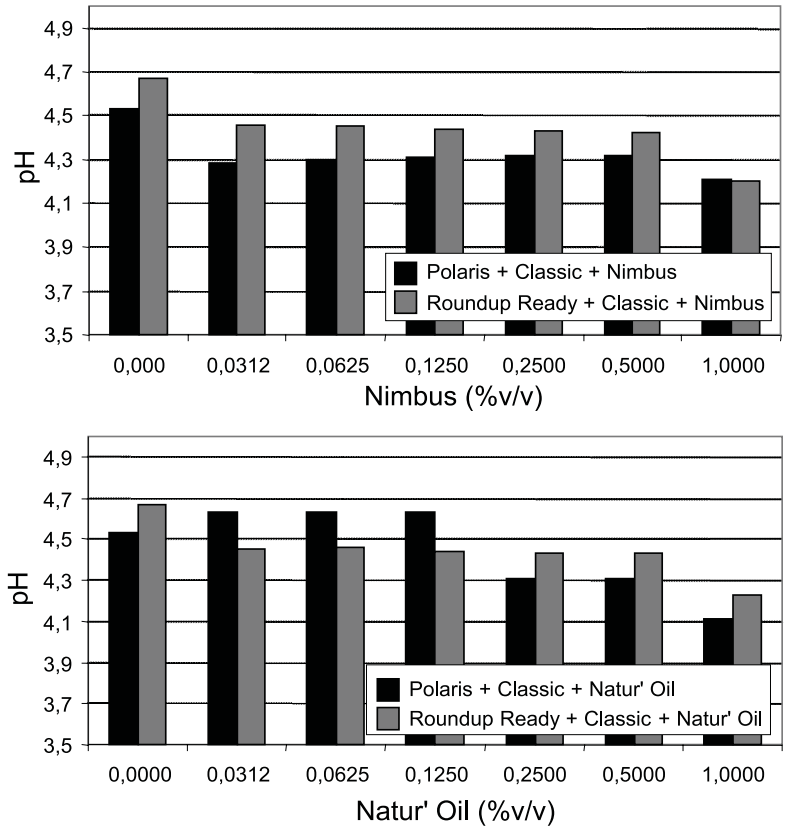

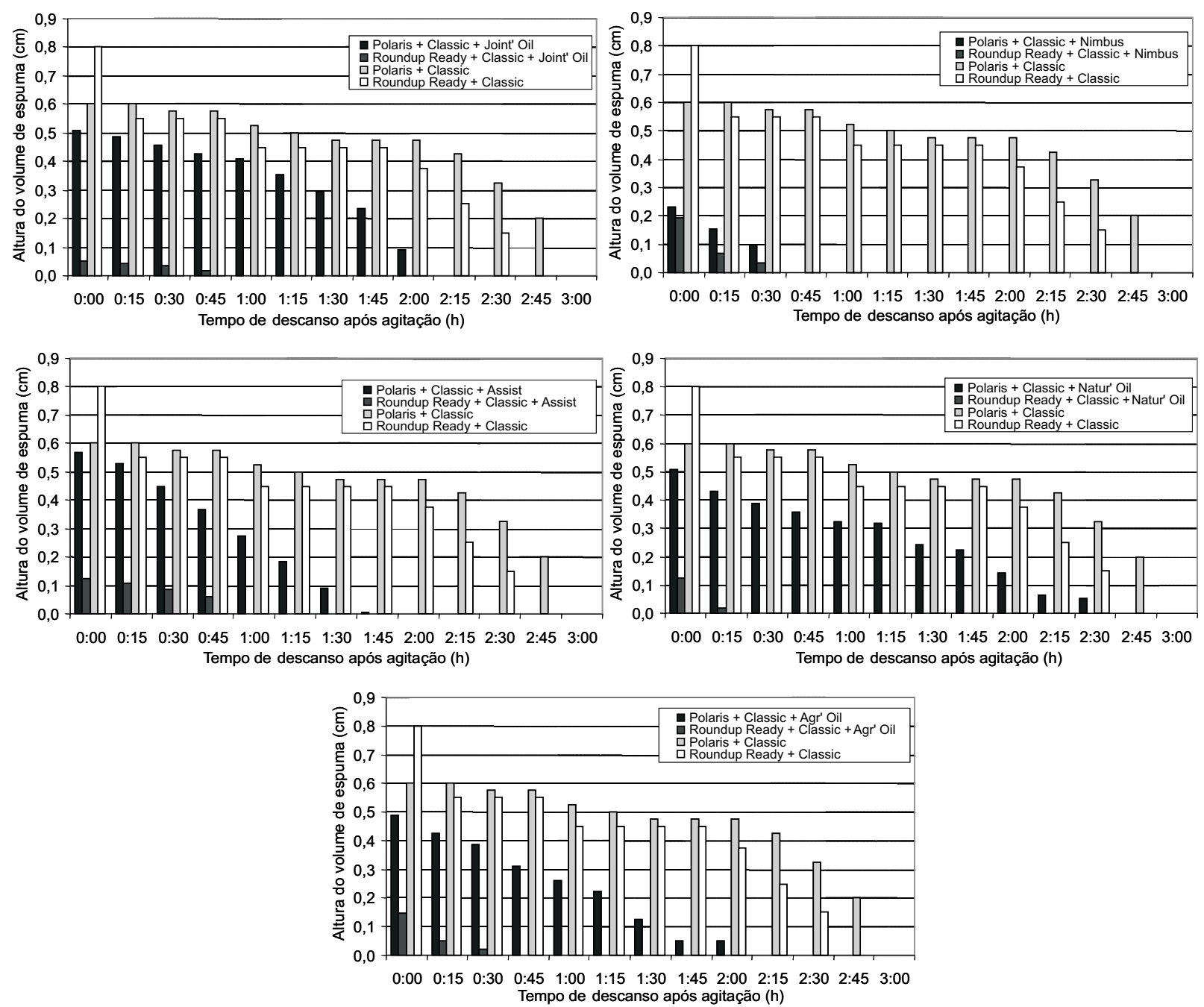

Figura 6 - Altura e estabilidade média do volume de espuma produzida após agitação de mistura em tanque dos herbicidas glyphosate $\left(\right.$ Polaris $^{\circledR}$ e Roundup Ready ${ }^{\circledR}$ ) e chlorimuron-ethyl $\left(\right.$ Classic $\left.^{\circledR}\right)$, associados com adjuvantes.

Com base nos resultados, pode-se afirmar que as misturas em tanque de chlorimuronethyl (Classic $\left.{ }^{\circledR}\right)$ com as formulações de glyphosate Polaris ${ }^{\circledR}$ e Roundup Ready ${ }^{\circledR}$ não interferiram nas características físicoquímicas de tensão superficial estática e pH das soluções, apesar de apresentarem maior quantidade e persistência de espuma, após o preparo da calda de pulverização. Todos os adjuvantes promoveram pequenas variações dos niveis médios de $\mathrm{pH}$ das soluções (inferiores a 0,5), mas reduziram a tensão superficial e a quantidade e persistência da espuma quando associados às misturas de Polaris ${ }^{\circledR}+$ Classic $^{\circledR}$ e Roundup Ready ${ }^{\circledR}$ Classic $^{\circledR}$, com destaque para o óleo mineral
Nimbus ${ }^{\circledR}$. Entretanto, é importante ressaltar que no Brasil ainda são restritos os resultados de pesquisas referentes às características fisico-químicas entre adjuvantes e misturas em tanque com herbicidas, as quais podem estabelecer informações complementares para auxíliar no posicionamento de resultados de eficácia e seletividade para as mais diferentes culturas.

\section{LITERATURA CITADA}

BIANCO, C. A. Tensão superficial e estado físico. In: ENCONTRO NACIONAL DE FORMULAÇÕES DE DEFENSIVOS AGRÍCOLAS, 1., 1985, São Paulo. Anais... São Paulo: Instituto Biológico de São Paulo, 1985. p. 161-172. 
BUICK, R. D.; BUCHAN, G. D.; FIELD, R. J. The role of surface tension of spreading droplets in absorption of a herbicide formulation via leaf stomata. Pestic. Sci., v. 38, p. $227-235,1993$

CARBONARI, C. A. et al. Controle químico de Panicum repens e Paspalum repens. Planta Daninha, v. 22, n. 3, p. $453-460,2004$.

CHUNG, B. J.; KWON, Y. W. Relationship between surfactant properties and wettability of rice leaf surfaces for several nonionic surfactants. In: FOY, C. L. (Ed.) Adjuvants for agrochemicals. Boca Raton: CRC Press, 1992. p. 37-58.

COSTA, N. V. et al. PH foliar e deposição de gotas de pulverização em plantas daninhas aquáticas: Brachiaria mutica, Brachiaria subquadripara e Panicum repens Planta Daninha, v. 23, n. 2, p. 295-304, 2005.

COUPLAND, D.; ROBINSON, S. The evaluation of ten emulsifiers for use with a mineral oil adjuvant. In: FOY, C. L. (Ed.) Adjuvants for agrochemicals. Boca Raton: CRC Press, 1992. p. 449-461

CUNHA, J. P. A. R. et al. Avaliação de estratégias para redução da deriva de agrotóxicos em pulverizações hidráulicas. Planta Daninha, v. 21, n. 2, p. 325-332, 2003.

DAN, H. A. et al. Efeito do pH da calda de pulverização na dessecação de Brachiaria brizantha com o herbicida glyphosate. Sci. Technol., v. 2, n. 1, p. 1-6, 2009.

ELLIS, M. C .B.; TUCK, C. R. The effect liquid properties on the spray formulations by flat fan nozzles. Aspect Appl. Biol., v. 48, n. 1 , p. $105-112,1997$.

EMPRESA BRASILEIRA DE PESQUISA AGROPECUÁRIA - EMBRAPA. Centro Nacional de Pesquisas de Solos. Manual de métodos de análises de solo. Rio de janeiro: 1997. 212 p.

FERREIRA, E. A. et al. Composição química da cera epicuticular e caracterização da superfície foliar em genótipos de cana-de-açúcar. Planta Daninha, v. 23,n. 4, p. 611-619, 2005.

MATUO, T.; BABA, K. J. Retenção de líquidos pelas folhas de citros em pulverização a alto volume. Científica, v. 9, n. 1, p. 97-104, 1981.

McCORMICK, R. W. Effects of $\mathrm{CO}_{2}, \mathrm{~N}_{2}$, air and nitrogen salts on spray solution pH. Weed Technol., v. 4, n. 4, p. 910-912, 1990.

MENDONÇA, C. G. et al. Efeito de surfatantes sobre a tensão superficial e a área de molhamento de soluções de glyphosate sobre folhas de tiririca. Planta Daninha, v. 17, n. 3, p. 355-365, 1999.

Planta Daninha, Viçosa-MG, v. 28, n. 3, p. 673-685, 2010
MENDONÇA, C. G. et al. Volume de espuma e estabilidade de emulsões aquosas com óleos minerais e óleos vegetais. In: SIMPÓSIO INTERNACIONAL DE TECNOLOGIA DE APLICAÇÃO DE AGROTÓXICOS, 3., 2004, Botucatu. Anais... Botucatu: FEPAF, 2004. p. 264-267.

MILLER, P. C. H.; BUTLER ELLIS, M. C. Effects of formulation on spray nozzle performance for applications from ground-based boom sprayers. Crop Protec., v. 19, n. 8-9, p. $609-615,2000$

MONTÓRIO, G. A. Eficiência dos surfatantes de uso agrícola na redução da tensão superficial. 2001. $70 \mathrm{f}$. Dissertação (Doutorado em Agronomia - Proteção de Plantas) - Universidade Estadual Paulista, Botucatu, 2001.

MONQUERO, P. A. et al. Caracterização da superfície foliar e das ceras epicuticulares em Commelina benghalensis, Ipomoea grandifolia e Amaranthus hybridus. Planta Daninha, v. 22, n. 2, p. 203-210, 2004.

PROCÓPIO, S. O. et al. Anatomia foliar de plantas daninhas do Brasil. Viçosa, MG: Universidade Federal de Viçosa, 2003. v. 1.118 p.

ROMAN, E. S. et al. Como funcionam os herbicidas: da biologia à aplicação. Passo Fundo: Berthier, 2007. 160 p.

ROSEN, M. J. Surfactants and interfacial phenomena 2.ed. New York: John Wiley \& Sons, 1989. 423 p.

SANCHOTENE, D. M. et al. Influência de sais e do pH da água na eficiência de imazethapyr + imazapic no controle de arroz-vermelho. Planta Daninha, v. 25, n. 2, p. 415-419, 2007.

SCHÖNHERR, J. et al. Foliar uptake of pesticides and its activation by adjuvantes: Theories and methods for optimization. In: INTERNATIONAL CONGRESS OF

PESTICIDE CHEMISTRY: ADVANCES IN INTERNATIONAL RESEARCH, DEVELOPMENT, AND LEGISLATION, 7., 1990, Hamburgo.

Proceedings...Weinheeim: VCH, 1991. p. 237-253.

SHERRICK, S. L.; HOLT, H. A.; HESS, F. D. Effects of adjuvants and environment during plant development on glyphosate absorption and translocation in field bindweed (Convolvulus arvensis). Weed Sci., v. 34, n. 6, p. 811-816, 1986.

SINGH, M.; MARCK, R. E. Effect of organosilicone-based adjuvants on herbicide efficacy. Pestic. Sci., v. 38, n. 2/3, p. $219-225,1993$

SILVA, F. M. L.; VELINI, E. D.; CORRÊA, T. M. Influência dos íons $\mathrm{Mg}$, Ca, Fe, Cu e Zn sobre a tensão superficial estática de soluções contendo surfatante. Planta Daninha, v. 24 , n. 3 , p. $589-595,2006$. 
SILVA, A. R.; LEITE, M. T.; FERREIRA, M. C. Estimativa da área foliar e capacidade de retenção de calda fitossanitária em cafeeiro. Biosci. J., v. 24, n. 3, p. 66-73, 2008.

THEISEN, G.; RUEDELL, J.; BIANCHI, M. A. Tecnologia de aplicação de herbicidas: teoria e prática. In: THEISEN, G.; RUEDELL, J. (Eds.). Aspectos técnicos da aplicação de herbicidas. Cruz Alta: Aldeia Norte, 2004. p. 25-54
THEISEN, G.; RUEDELL, J. Tecnologia de aplicação de herbicidas: teoria e prática. Passo Fundo: Aldeia Norte, 2004. $90 \mathrm{p}$.

VELINI, E. D.; MARTINS, D.; SILVA, M. A. S. Efeito de concentração de espalhante adesionante e doses de glyphosate no controle de Brachiaria decumbens e Panicum maximum Planta Daninha, v. 18, n. 2, p. 349-366, 2000 